\title{
An Estimator of the Resistance of Large Grounding Electrodes from Its Geometric Characterization
}

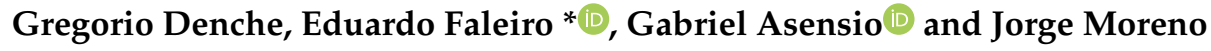 \\ Escuela Técnica Superior de Ingeniería y Diseño Industrial (ETSIDI), Polytechnic University of Madrid (UPM), \\ Ronda de Valencia 3, 28012 Madrid, Spain; gregorio.denche@upm.es (G.D.); gabriel.asensio@upm.es (G.A.); \\ jorge.moreno@upm.es (J.M.) \\ * Correspondence: eduardo.faleiro@upm.es
}

Received: 30 September 2020; Accepted: 16 November 2020; Published: 18 November 2020

\begin{abstract}
An estimator of the grounding resistance of large extension electrodes from the total length and geometric properties of the electrode is proposed in this work. The approximation is valid for electrodes buried in a soil that can be assumed as homogeneous and it will be verified that the resistance estimation improves as electrode size increases. Both the burial depth and the radius of the conductors, within certain practical limits, have little effect on the value of the grounding resistance. The shape and size of the electrode are defined by a geometric index that measures the degree of compaction of the conductors that make it up, which has the greatest weight in the final value of the resistance. The expression obtained to quantify the estimate of the grounding resistance is tested in several numerical examples and in a currently operating grounding electrode, obtaining in all cases an estimated value of the resistance reasonably close to the true value.
\end{abstract}

Keywords: grounding resistance estimator; large grounding electrodes; stretching index

\section{Introduction}

Determining the theoretical value of the grounding resistance of an electrode is a well-known calculation process that requires knowledge of the geometry of the conductors that make up the electrode, their location in the ground, burial depth, orientation and of the conductive soil model. The electrode must be composed by conductors in the form of thin wires, which must be segmented in the calculation process to build the impedance matrix from which, by an inversion procedure, the distribution of leakage currents to the ground is obtained by all the segments into which the entire electrode has been divided [1-3]. The calculation is made possible by imposing either the electrode to be equipotential if the conductor is considered perfect, or the distribution of potentials according to the entry point of the total current to be filtered to the ground in the case of real conductors with non-zero internal resistivity [4]. As the electrode grows in size, the impedance matrix gets bigger and bigger with the consequent computational cost to carry out the matrix inversion. A thicker segmentation can help to speed up the calculation, but in very large electrodes this can lead to obtaining theoretical resistance values that could be far from the true value although always above it [5].

For decades, some authors have worked on finding calculation expressions for the grounding resistance of an electrode without carrying out the direct calculation based on the determination of the potential acquired by the electrode itself by means of numerical techniques. Many of these works are focused on grounding grids for substations. Some use properties of these electrodes such as the duality between resistance and capacitance [6]. Others, on the other hand, deal with a calculation of $R$ based on the equipotential surfaces of the conductive soil around the electrode, which are evaluated using FEM [7]. Another group of works base their estimators on empirical expressions for simple structures, which combined with each other, build a complex electrode [8]. 
On the other hand, it is not easy to find research that relates the grounding resistance with the geometric properties of the electrodes. In the field of geophysics, mainly related to electrode arrays in electric resistivity tomography (ERT), some work can be found in which an attempt is made to establish a relationship between geometric configurations of electrodes with the electric resistance in order to improve geoelectric measurements of the soil [9].

In the present work, an expression is proposed to estimate the grounding resistance of an electrode buried in a homogeneous soil, which is based on a known fact, although scarcely cited in the literature. An electrode of total length $L$ presents a grounding resistance that is so much lower the more stretched it is, in other words, the further away some parts of the electrode are from others of the electrode itself. The minimum resistance is only achieved by completely stretching the electrode in a straight line. This property is nothing more than a consequence of the interaction between conductors, which causes the potential to decrease with the distance between them [10]. An electrode of length $L$ folded has a higher grounding resistance than the same electrode stretched in a straight line. If the degree of stretching of an electrode, here quantified by the so-called stretching index $i_{s}$ of the electrode, is defined so that it is independent of the size of the electrode and the segmentation used to divide it, a relationship could be established between the grounding resistance $R$, the length $L$ of the electrode and the stretch index $i_{s}$. This relationship depends, in principle, on the burial depth $h$, on the radius $r$ of the thin wire conductors that make up the electrode and on the homogeneous resistivity of the ground $\rho$. However, it is known that the dependence of $\mathrm{R}$ with $\mathrm{h}$ and $\mathrm{r}$ attenuates as the length of the conductor increases, so the estimate of $\mathrm{R}$ proposed here will give better results as the electrode length L increases.

To determine the relationship between $\mathrm{R}, \mathrm{L}$ and $\mathrm{i}_{\mathrm{s}}$, we will proceed through numerical experiments considering electrodes of various lengths within a wide range. For each length, several bends of the electrode will be considered, from the rectilinear configuration with the highest possible stretching index without breaking the electrode into disjoint parts, to a folding with the shape of the letter $\mathrm{M}$, where the stretching index is low, due to the proximity of some parts of the electrode with others of the electrode itself. With the data obtained from the numerical experiments, the desired relationship will be obtained, the robustness of which will be tested by varying the conditions of the experiments that involve the parameters $h$ and $r$, checking their scarce relevance in the final result.

To achieve these objectives, this paper is structured as follows. After the present introduction, in Section 2 the concept of stretching index $i_{s}$ is introduced, proposing the necessary numerical experiments to establish the relationship between $\mathrm{R}, \mathrm{L}$ and $\mathrm{i}_{\mathrm{s}}$, which will be quantitatively proposed. In Section 3 some tests are carried out on synthetic electrodes in order to validate the proposed expression. In the same section an estimate is obtained on a real and operational large electrode whose grounding resistance is known, for a final validation of the proposed expression. Finally, Section 4 presents the conclusions of the present work.

\section{Basics of the Method}

As mentioned in the introduction, an electrode of length $L$ buried in a conductive soil and excited by a current I, presents an electrical resistance that depends significantly on its geometric shape. As the electrode is stretched its resistance decreases. This fact is explained by the decrease (increase) in the electric potential of the mutually interacting electrodes when they move away (get closer). To quantify how packed or stretched its profile is, the so-called stretching index $i_{s}$ is introduced here, which is defined by the following relationship

$$
\mathrm{i}_{\mathrm{S}}=\frac{\sum_{\mathrm{i}, \mathrm{j} \geq \mathrm{i}=1}^{\mathrm{N} \cdot \mathrm{L}}\left|\overrightarrow{\mathrm{r}}_{\mathrm{i}}-\overrightarrow{\mathrm{r}}_{\mathrm{j}}\right|}{\mathrm{N}^{2} \mathrm{~L}^{3}}
$$


where $\mathrm{N}$ is the number of segments per meter of conductor, $\mathrm{L}$ is the total length of the electrode and $\left|\vec{r}_{i}-\vec{r}_{j}\right|$ is the distance between segment $i$ and segment $j$. From Equation (1) it is observed that a stretched electrode will present an index $i_{s}$ greater than a more compacted one of the same length and number of segments per meter. The dimensionless property of Equation (1) guarantees that $i_{s}$ will not change when considering equally stretched electrodes but of different $\mathrm{L}$ and $\mathrm{N}$.

In order to establish a functional relationship between the grounding resistance $\mathrm{R}$, the total length of the electrode $\mathrm{L}$ and the stretching index $i_{s}$, numerical experiments with electrodes of lengths $\mathrm{L}=1$ $\mathrm{m}, 4 \mathrm{~m}, 7 \mathrm{~m}, 10 \mathrm{~m}$ and $20 \mathrm{~m}$ will be carried out. For each length, several geometric shapes of different folding will be considered to numerically establish the relationship $R=f\left(L, i_{s}\right)$. In these experiments a current $\mathrm{I}=1 \mathrm{~A}$ will be injected to the electrodes, which are buried in a homogeneous soil with resistivity $\rho=1 \Omega \mathrm{m}$. The electrodes are made up of perfect conductive thin wires of a radius set to $\mathrm{r}=6 \mathrm{~mm}$ and are assumed to be buried horizontally at a depth $h=0.5 \mathrm{~m}$. For each electrode considered with a length and shape preset, the grounding resistance is calculated by means of the boundary elements method combined with the moments method using a code written by the authors [11], which is inspired by works such as those already cited in the introduction. In a very concise way, the theoretical calculation of the resistance is carried out by calculating the potential acquired by the electrode with respect to the potential of infinity, which is assumed to be zero. Regarding the numerical calculation of the potential, the electrode is divided into smaller portions that filter a part of the total injected current to the ground so that between they all generate a total potential that is the same in all those conductor portions. This procedure leads to a linear system of equations from which the currents filtered by all the portions and also the common potential of all of them, which is the potential of the electrode itself, are obtained. For different soil resistivity and current intensity, R has to be multiplied by the factor $\rho \mathrm{I}$, while the dependence of $R$ with $r$ and $h$ attenuates as $L$ increases, as will be seen later.

The geometric shapes considered here are shown in Figure 1. The common length of all these shapes is $\mathrm{L}$ and they range from the straight line, with the maximum stretching, to the capital letter $\mathrm{M}$ shape, with the minimum stretching in the series. Except for the rectilinear shape, the total length $\mathrm{L}$ has been divided into rods of equal size.
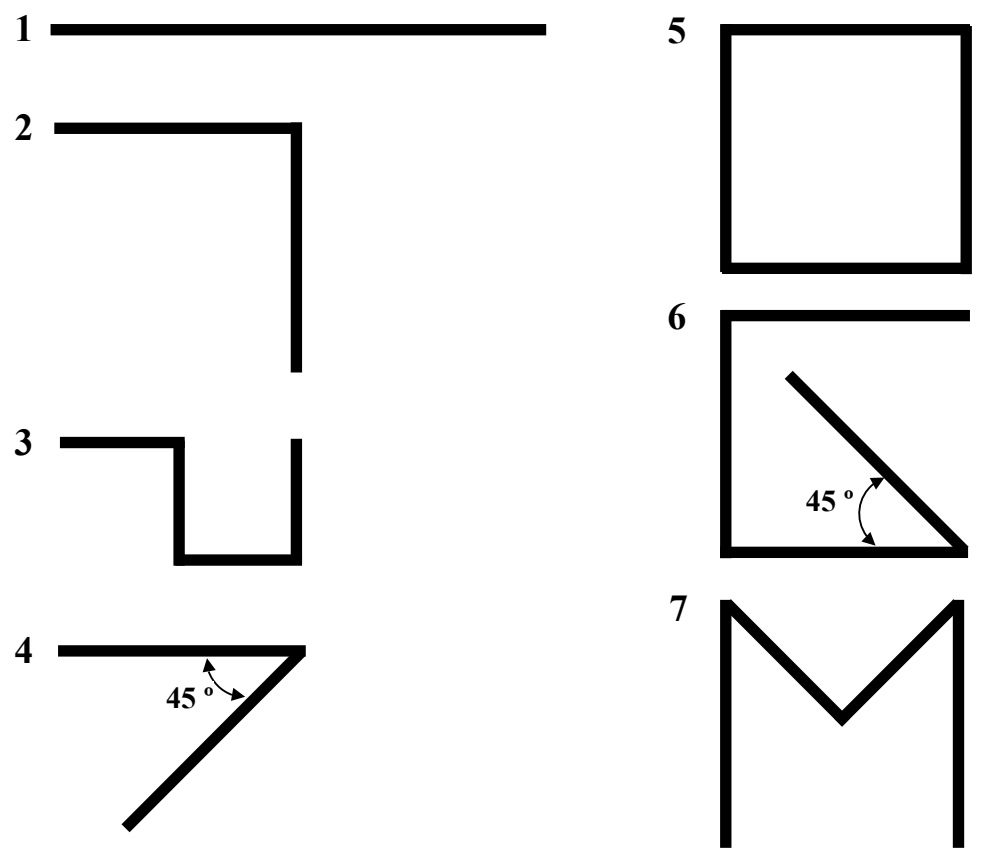

Figure 1. Electrode shapes numbered from 1 to 7 , with common length $\mathrm{L}$ and sorted by the stretching index $i_{s}$. 
The theoretical calculation of the resistance was carried out using the boundary elements method together with the moments method to obtain a linear numerical approximation to the problem $[2,3]$. Table 1 shows the results of the numerical experiments with the electrodes whose geometric shapes are shown in Figure 1. The five total lengths $L$ mentioned above are considered, for which the resistance $\mathrm{R}$ was calculated. For all calculations, segmentation was set to $\mathrm{N}=20$ segments per meter of conductive wire.

Table 1. Grounding resistance $\mathrm{R}$ in Ohms for all types of electrodes in Figure 1 as a function of the index $i_{s}$ and the electrode length $L$, when the current is $I=1 \mathrm{~A}$ and the soil resistivity is $\rho=1 \Omega \mathrm{m}$. The segmentation is stated at $\mathrm{N}=20$ segments $/ \mathrm{m}$.

\begin{tabular}{ccccccccccc}
\hline & \multicolumn{2}{c}{$\mathbf{L}=\mathbf{1} \mathbf{~ m}$} & \multicolumn{2}{c}{$\mathbf{L}=\mathbf{4} \mathbf{~ m}$} & \multicolumn{2}{c}{$\mathbf{L}=\mathbf{~} \mathbf{m}$} & \multicolumn{2}{c}{$\mathbf{L}=\mathbf{1 0} \mathbf{~}$} & \multicolumn{2}{c}{$\mathbf{L}=\mathbf{2 0} \mathbf{~ m}$} \\
\cline { 2 - 11 } & $\mathbf{i}_{\mathbf{s}}$ & $\mathbf{R}$ & $\mathbf{i}_{\mathbf{s}}$ & $\mathbf{R}$ & $\mathbf{i}_{\mathbf{s}}$ & $\mathbf{R}$ & $\mathbf{i}_{\mathbf{s}}$ & $\mathbf{R}$ & $\mathbf{i}_{\mathbf{s}}$ & $\mathbf{R}^{*} \mathbf{1 0} \mathbf{- 1}$ \\
\hline $\mathbf{1}$ & 0.166 & 0.835 & 0.167 & 0.296 & 0.167 & 0.192 & 0.167 & 0.145 & 0.167 & 0.832 \\
\hline $\mathbf{2}$ & 0.137 & 0.864 & 0.137 & 0.307 & 0.137 & 0.199 & 0.138 & 0.150 & 0.138 & 0.859 \\
\hline $\mathbf{3}$ & 0.111 & 0.916 & 0.111 & 0.324 & 0.111 & 0.211 & 0.111 & 0.159 & 0.111 & 0.906 \\
\hline $\mathbf{4}$ & 0.107 & 0.945 & 0.108 & 0.327 & 0.108 & 0.212 & 0.108 & 0.160 & 0.108 & 0.913 \\
\hline $\mathbf{5}$ & 0.092 & 0.977 & 0.092 & 0.344 & 0.092 & 0.224 & 0.092 & 0.169 & 0.092 & 0.961 \\
\hline $\mathbf{6}$ & 0.082 & 1.017 & 0.083 & 0.355 & 0.083 & 0.232 & 0.083 & 0.175 & 0.083 & 0.997 \\
\hline $\mathbf{7}$ & 0.076 & 1.083 & 0.077 & 0.374 & 0.077 & 0.243 & 0.077 & 0.184 & 0.077 & 1.049 \\
\hline \multicolumn{4}{c}{ The header $\mathbf{R} * \mathbf{1 0}$ means that the column data should be divided by 10.} &
\end{tabular}

As previously stated, the stretching index $i_{s}$ remains constant for each geometric shape, regardless of its size.

From these data, a power law that relates $\mathrm{R}$ and $\mathrm{i}_{\mathrm{s}}$ for each value of $\mathrm{L}$ was proposed. The choice is motivated by the general form of any physical law, which, according to the dimensional analysis theory [12], is expressed as a power law between magnitudes. Equation (2) specifies the proposal.

$$
\mathrm{R}=\mathrm{R}_{\mathrm{L}} \mathrm{i}_{\mathrm{s}} \beta
$$

In this expression, $R_{L}$ is a multiplicative factor that could depend on $L$, on the soil resistivity $\rho$ and less sensitively on the burial depth $h$ and the radius of the conductors $r$. A new power law for $R_{L}$ is proposed here, $R_{L}=R_{0} \rho L^{\alpha}$, so that the electrode resistance $R$ will have the functional form

$$
\mathrm{R}=\mathrm{R}_{0} \rho \mathrm{L}^{\alpha} \mathrm{i}_{\mathrm{s}} \beta
$$

where $R_{0}$ is a constant whose value changes very little with $h$ and $r$ as $L$ increases, so it will take the value obtained from the calculations with $h=0.5 \mathrm{~m}$ and $\mathrm{r}=0.006 \mathrm{~m}$, values that can be considered typical of many grounding devices.

Table 2 shows the results of fitting the data in Table 1 to the power law $R=R_{L} i_{s}{ }^{\beta}$. As can be seen, $R_{L}$ shows a dependency with $L$ that fits quite well to the aforementioned law $R_{L}=R_{0} \rho L^{\alpha}$, as it is shown in Figure 2. 
Table 2. Numerical fitting to the power law Equation (2) from data of Table 1.

\begin{tabular}{ccc}
\hline $\mathbf{L}(\mathbf{m})$ & $\mathbf{R}_{\mathbf{L}}$ & $\boldsymbol{\beta}$ \\
\hline 1 & 0.456 & -0.324 \\
\hline 4 & 0.171 & -0.296 \\
\hline 7 & 0.110 & -0.302 \\
\hline 10 & 0.083 & -0.298 \\
\hline 20 & 0.046 & -0.314 \\
\hline
\end{tabular}

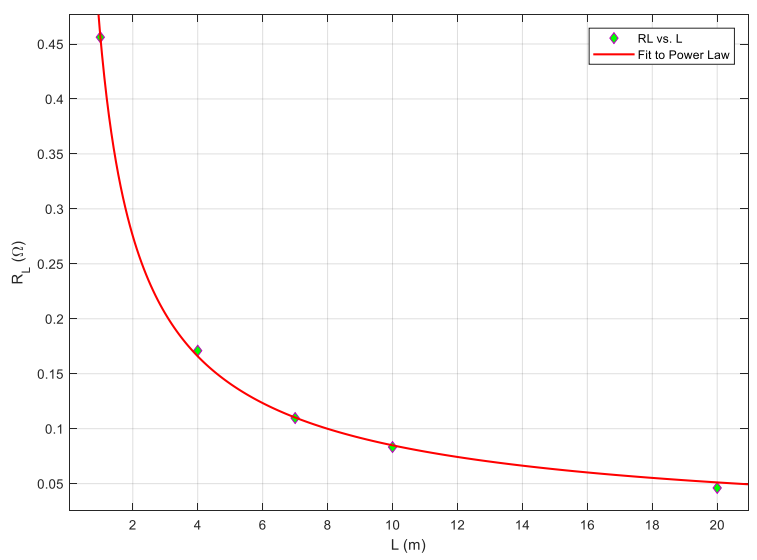

Figure 2. The power law $\mathrm{R}_{\mathrm{L}}$ vs. $\mathrm{L}$ when $\rho=1 \Omega \mathrm{m}$.

The exponent $\beta$ shows a certain dispersion, so the mean value of $\beta=-0.307$ and a $95 \%$ confidence interval [-0.319, -0.295$]$ will be taken for this parameter. Fitting to the power law $R_{L}=R_{0} \rho L^{\alpha}$ with $\rho=1 \Omega \mathrm{m}$, the results for the value of $\mathrm{R}_{0}$ and $\alpha$ parameters together with a $95 \%$ confidence interval are $\mathrm{R}_{0}=0.457 \pm 0.014$ and $\alpha=-0.731 \pm 0.038$, from which the desired relationship Equation (4) is obtained

$$
R=R_{0} \rho L^{\alpha} i_{s} \beta \quad\left\{\begin{array}{l}
R_{0}=0.457 \\
\alpha=-0.731 \\
\beta=-0.307
\end{array}\right.
$$

To justify that the dependence with $\mathrm{h}$ and $\mathrm{r}$ is not considered, a numerical experiment will be carried out in which the resistance of electrodes will be theoretically calculated as a function of the length $L$, burial depth $h$ and radius $r$. A horizontal rod type electrode of variable length $L$ with values $1,3,5,7,10,15,20$ and $30 \mathrm{~m}$ was considered. For each length, a range of values of the radius $r$ among 2 , $4,6,8$ and $10 \mathrm{~mm}$ were used and for each radius, a range of values of the burial depth $\mathrm{h}$ among $0.1,0.3$, $0.5,0.7$ and $0.9 \mathrm{~m}$ were taken into the calculations.

Figure 3 shows the variation of the grounding resistance $R$ as a function of the length of the electrode $L$ when $r$ varies for a fixed value of $h$, on the left panel, and when $h$ varies for a fixed value of $r$, on the right panel. From the mentioned figure, it follows that as the length $L$ of the electrode increases, the variation of $\mathrm{R}$ with $\mathrm{h}$ and $\mathrm{r}$ is clearly the more attenuated the longer the length of the electrode is.

Regarding the uncertainty in determining the estimator of $\mathrm{R}$, assuming no correlation between the variables $R_{0}, \alpha$ and $\beta$, the standard deviation of $R$ is calculated by

$$
\sigma_{\mathrm{R}}=\mathrm{R} \cdot \sqrt{\frac{\sigma_{\mathrm{R}_{0}}^{2}}{\mathrm{R}_{0}^{2}}+(\log (\mathrm{L}))^{2} \cdot \sigma_{\alpha}^{2}+\left(\log \left(\mathrm{i}_{\mathrm{s}}\right)\right)^{2} \cdot \sigma_{\beta}^{2}}
$$


where $\sigma_{i}^{2}$ are the variances of the $R_{0}, \alpha$ and $\beta$ variables. From Equation (5), a 95\% confidence interval could be obtained for the estimator of $R$ by $\left[R-Z \frac{\sigma_{R}}{\sqrt{N}}, R+Z \frac{\sigma_{R}}{\sqrt{N}}\right]$ with $Z=1.96$.

As a conclusion, it can be stated that Equation (4) can be accepted as an approximation to the resistance with better results as the length of the electrode increases. The possible variation of the numerical parameters of Equation (4) with the burial depth $h$ and the electrode radius $r$ can be ignored, always within the limits imposed by practical applications.
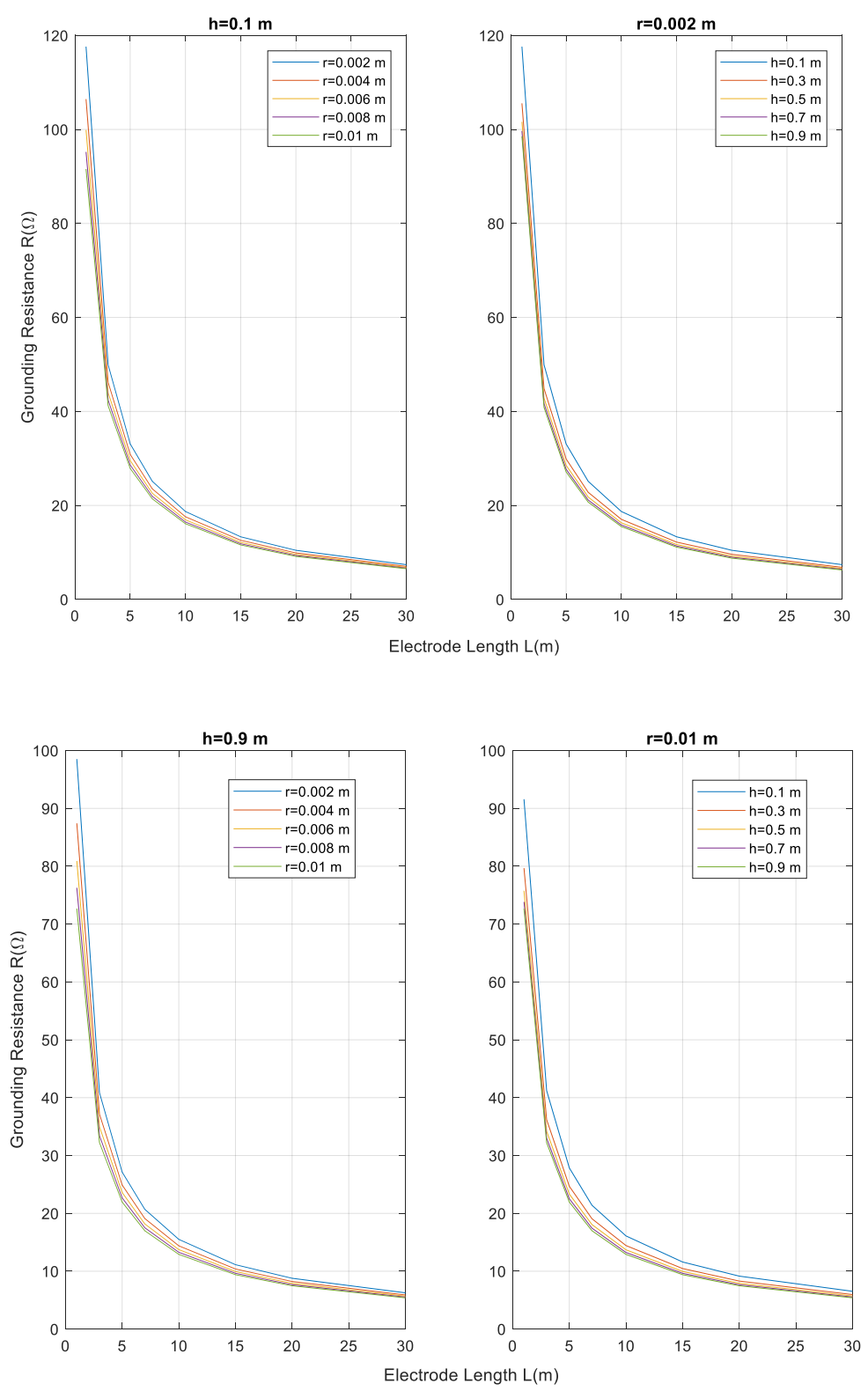

Figure 3. Grounding resistance vs. the electrode length $\mathrm{L}$ when $\mathrm{h}$ and $\mathrm{r}$ take extreme values according to their ranges of variation, as indicated in the text.

\section{The Estimation of $R$ on Synthetic Models. Estimation of the Resistance of a Real Operating Electrode}

In this section, Equation (4) will be tested on some electrode models already used and on other electrodes that will be fully defined in the text.

As a first test, the result of the estimation of the resistance $R$ of the type 7 electrode, with a total length of $\mathrm{L}=20 \mathrm{~m}$ and with a segmentation of $\mathrm{N}=20$ segments per meter in a soil of resistivity 
$\rho=1 \Omega \mathrm{m}$, was tested. The result of the estimation was $\mathrm{R}=(0.112 \pm 0.014) \Omega$ while the theoretically calculated one was $R=0.105 \Omega$, which represents a percentage difference of less than $7 \%$ between them. However, the confidence interval of the estimate contained the theoretically calculated value.

As a second test of the estimation formula Equation (4), a large square electrode as a regular grid of a $100 \mathrm{~m}$ side and composed by 840 rods of $5 \mathrm{~m}$ long and $4 \mathrm{~mm}$ radius, will be considered. The electrode is buried at $0.5 \mathrm{~m}$ in a homogeneous soil of $\rho=100 \Omega \mathrm{m}$ resistivity, so that the theoretical calculation is not computationally very expensive, since it involves a considerable number of rods, the segmentation is set at 2 segments per rod, clearly poor but necessary if we want the theoretical calculation to be carried out in a reasonable time. This calculation results in $R=0.448 \Omega$. Estimation through Equation (4) requires the calculation of $i_{S}$ with $N=2 / 5$ segments $/ \mathrm{m}$ or any other segmentation and $L=4200 \mathrm{~m}$. This is done in a simple and very quick way, resulting in $\mathrm{i}_{\mathrm{s}}=0.0064$. Finally, the estimation from the Equation (4) results in $R=(0.391 \pm 0.126) \Omega$, which represents a percentage difference of around $13 \%$ between the representative values, although it is observed that the calculated value was within the confidence interval of the estimator. The difference was large, but taking into account that $i_{\mathrm{s}}$ will not change in value if the segmentation is refined and that an increase in segmentation always produces a decrease in the theoretically calculated resistance, we could conclude that this percentage difference will decrease if a more precise theoretical calculation, with a greater segmentation, of the electrode resistance is carried out.

Next, the estimate of the grounding resistance of a real operating electrode will be obtained from Equation (4). Specifically, the resistance of the grounding electrode of the Balaidos electrical substation located in Vigo, Galician Community (Spain), will be estimated. Figure 4 shows the geometric structure of the electrode, which is a non-regular grid formed by rods of variable length with a total conductor length of $289.77 \mathrm{~m}$.

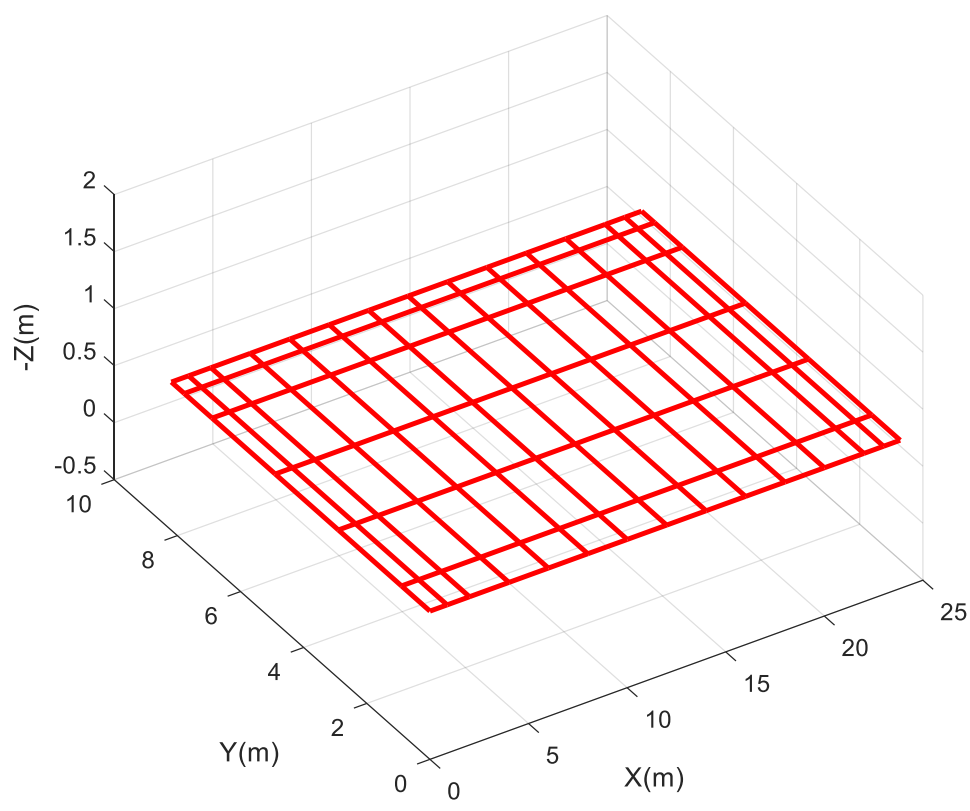

Figure 4. The Balaidos substation grounding grid.

The electrode is buried at a depth of $0.8 \mathrm{~m}$ and the parameter $\mathrm{k}_{\mathrm{R}}=\frac{\mathrm{R}}{\rho}$ is previously known. Its value is $k_{R}=0.028 \Omega / \Omega \mathrm{m}$, which represents the grounding resistance of the electrode, buried in a homogeneous soil of resistivity $\rho=1 \Omega \mathrm{m}$. The resistance estimated by Equation (4) for a soil of such a resistivity with a segmentation of $\mathrm{N}=2.5$ segments $/ \mathrm{m}$ and a stretching index of $\mathrm{i}_{\mathrm{s}}=0.030$ resulted in a value of $R=(0.021 \pm 0.005) \Omega$, an interval whose upper end almost reached the real value that the electrode would have. 
In summary, the estimation of $\mathrm{R}$ for a specific electrode requires knowledge of its total length $\mathrm{L}$, and its precise geometric shape in order to calculate $i_{s}$, just the same information needed to theoretically calculate the resistance. With this information available, a suitable segmentation as a choice of $\mathrm{N}$ segments per meter of straight conductor can be carried out and thus determine the stretching index $\mathrm{i}_{\mathrm{s}}$ by simple calculation. Equation (4) was then applied and the estimate of $R$ was thus obtained.

As a final comment, the numerical values of the constants that appear in Equation (4) can be refined from the knowledge of the resistance of other electrodes than those used here for the fitting, which has led to Table 2. In this way, by having a larger number of data available to determine the fitting parameters of Equation (4), the confidence intervals could be reduced, gaining in the estimator precision.

\section{Conclusions}

In this work, an estimator of the grounding resistance of horizontal electrodes buried in a homogeneous soil was proposed. The estimator was more accurate as the electrode got bigger. Complete information on the geometric shape of the electrode and its total length is necessary. By calculating the stretching index for which segmentation should be chosen, the application of Equation (4) leads to a value of the estimate together with its confidence interval. The estimator was tested on various synthetic electrodes and on a real electrode of known grounding resistance, with good results.

The stretching index $i_{s}$ was easy and quick to calculate from the electrode geometric shape and thus Equation (4) could provide a first approximation to the resistance of an electrode in the design stage of a grounding system. The usefulness of an estimator like the one presented is reinforced when dealing with large electrodes, for which a theoretical calculation is sometimes very difficult to implement due to computational costs. Finally, the estimator can be improved if new information about electrodes other than those already used here can be added to improve the parameters of the fittings done also reducing their confidence intervals.

Author Contributions: All authors are involved to some extent in the different parts of the paper. In particular, G.D. and E.F. have established the foundations of the paper and designed the necessary software for calculations, J.M. and G.A. have contributed in a special way in theoretical calculations of the grounding resistance of large electrodes. All authors have read and agreed to the published version of the manuscript.

Funding: This research received no external funding.

Acknowledgments: The authors would like to thank both the Department of Applied Mathematics and the IEEF Department of the Escuela Técnica Superior de Ingeniería y Diseño Industrial (ETSIDI) at the Polytechnic University of Madrid (UPM) for their support to the undertaking of the research summarized here. Furthermore, the authors appreciate the collaboration with the firm INGESCO Ligthning Solutions at Terrassa, Barcelona (Spain), for the technical support and useful suggestions for this work.

Conflicts of Interest: The authors declare no conflict of interest

\section{References}

1. Garrett, D.L.; Holley, H.J. Calculation of substation grounding system resistance using matrix techniques. IEEE Trans. Power Appar. Syst. 1980, PAS-99, 2008-2011. [CrossRef]

2. Colominas, I.; Navarrina, F.; Casteleiro, M. A boundary element numerical approach for grounding grid computation. Comput. Methods Appl. Mech. Eng. 1999, 174, 73-90. [CrossRef]

3. Berberović, S.; Haznadar, Z.; Stih, Z. Method of moments in analysis of grounding systems. Eng. Anal. Bound. Elem. 2003, 27, 351-360.

4. Ma, X.; Cui, X.; Zhang, L.; Yang, H.; Yuan, J. Simulation of substation grounding grids with unequal-potential. IEEE Trans. Magn. 2000, 36, 1468-1471.

5. Freschi, F.; Mitolo, M.; Tartaglia, M. An Effective Semianalytical Method for Simulating Grounding Grids. IEEE Trans. Ind. Appl. 2013, 49, 256-263. [CrossRef]

6. Chow, Y.; Salama, M. A simplified method for calculating the substation grounding grid resistance. IEEE Trans. Power Deliv. 1994, 9, 736-742. [CrossRef] 
7. Guemes-Alonso, J.A.; Hernando-Fernandez, F.; Rodriguez-Bona, F.; Ruiz-Moll, J. A Practical Approach for Determining the Ground Resistance of Grounding Grids. IEEE Trans. Power Deliv. 2006, 21, 1261-1266. [CrossRef]

8. Trifunović, J.; Kostic, M.B. An Algorithm for Estimating the Grounding Resistance of Complex Grounding Systems Including Contact Resistance. IEEE Trans. Ind. Appl. 2015, 51, 5167-5174. [CrossRef]

9. Ingeman-Nielsen, T.; Tomaskovicova, S.; Dahlin, T. Effect of electrode shape on grounding resistances-Part 1: The focus-one protocol. Geophysics 2016, 81, WA159-WA167. [CrossRef]

10. Herrera, W.J.; Diaz, R.A. The geometrical nature and some properties of the capacitance coefficients based on Laplace's equation. Am. J. Phys. 2008, 76, 55-59. [CrossRef]

11. Faleiro, E.; Pazos, F.J.; Asensio, G.; Denche, G.; Garcia, D.; Moreno, J. Interaction Between Interconnected and Isolated Grounding Systems: A Case Study of Transferred Potentials. IEEE Trans. Power Deliv. 2014, 30, 2260-2267. [CrossRef]

12. Cheng, Y.-T.; Cheng, C.-M. Scaling, dimensional analysis, and indentation measurements. Mater. Sci. Eng. R Rep. 2004, 44, 91-149. [CrossRef]

Publisher's Note: MDPI stays neutral with regard to jurisdictional claims in published maps and institutional affiliations.

(C) 2020 by the authors. Licensee MDPI, Basel, Switzerland. This article is an open access article distributed under the terms and conditions of the Creative Commons Attribution (CC BY) license (http://creativecommons.org/licenses/by/4.0/). 